\title{
Low-energy $D^{*+} \bar{D}_{1}^{0}$ scattering and the resonance-like structure $Z^{+}(4430)$
}

\section{Gong ${ }^{* ;}$ G.-Z. Meng, S. He, C. Liu, Z.-Y. Niu, Y. Shen}

School of Physics, Peking University

E-mail: gongming@pku.edu.ch, gzhmeng@gmail.com, songracs@gmail.com

liuchuan@pku.edu.cn niuzy@pku.edu.cn shendandan@gmail.com

\section{Y. Chen, G. Li, Y.-J. Zhang}

Institute of High Energy Physics, Academia Sinica

E-mail: cheny@ihep.ac.cn, gli@mail.ihep.ac.cn,

yjzhang@mail.ihep.ac.cn

\section{Y.-B. Liu, X.-F. Meng}

Department of Physics, Nankai University

E-mail: liuyb@nankai.edu.cn, mengxf@mail.nankai.edu.cn

\section{J.-P. Ma}

Institute of Theoretical Physics, Academia Sinica

E-mail: majpeitp.ac.cn

\section{J.-B. Zhang}

Department of Physics, Zhejiang University

E-mail: jbzhang@zimp.zju.edu.cn

\begin{abstract}
Low-energy scattering of $D^{*}$ and $\bar{D}_{1}$ meson are studied using quenched lattice QCD with improved lattice actions on anisotropic lattices. The calculation is performed within Lüscher's finitesize formalism which establishes the relation between the scattering phase in the infinite volume and the exact energy level in the finite volume. We obtain the scattering length $a_{0}=2.52(47) \mathrm{fm}$ and the effective range $r_{0}=0.7(1) \mathrm{fm}$ in $J^{P}=0^{-}$channel. Based on these results, it is argued that, albeit the interaction between the two charmed mesons being attractive, it is unlikely that they can form a shallow bound state in this channel. This calculation provides some useful information on the nature of the newly discovered resonance-like structure $Z^{+}(4430)$ by the Belle Collaboration.
\end{abstract}

The XXVII International Symposium on Lattice Field Theory

July 26-31, 2009

Peking University, Beijing, China

\footnotetext{
*Speaker.

${ }^{\dagger}$ For the CLQCD Collaboration
} 


\section{Introduction}

Recently, a charged resonance-like structure $Z^{+}(4430)$ has been observed at Belle in the $\pi \psi^{\prime}$ invariant mass spectrum of $B \rightarrow K \pi^{+} \psi^{\prime}$ decays [1]. This discovery has triggered many theoretical investigations on the nature of this structure [2, 3, , , , 5, 6, 7, 8, 9]. Since the invariant mass of the resonance is very close to the $D^{*} \bar{D}_{1}$ threshold, one possible interpretation is a molecular bound state formed by the $D^{*}$ and $\bar{D}_{1}$ mesons [3]. To further investigate this possibility, the interaction between $D^{*}$ and $\bar{D}_{1}$ mesons becomes crucial. As is known, the interaction of two hadrons can be studied via the scattering process of the hadrons. Since the energy being considered here is very close to the threshold of the $D^{*} \bar{D}_{1}$ system, only threshold scattering parameters, i.e. scattering length $a_{0}$ and effective range $r_{0}$, are relevant. Here we briefly report our results obtained from a quench lattice QCD study using improved actions on anisotropic lattices [10].

\section{Strategies for the computation}

\subsection{Lüscher's finite volume technique and its generalization}

Within Lüscher's formalism [11, 12], the exact energy eigenvalue of a two-particle system in a finite box of size $L^{3} \times T$ is related to the elastic scattering phase of the two particles in the infinite volume. We define a variable $\overline{\mathbf{k}}^{2}$ for each diagonalized momentum mode via:

$$
E_{i}=\sqrt{m_{D^{*}}^{2}+\overline{\mathbf{k}}^{2}}+\sqrt{m_{D_{1}}^{2}+\overline{\mathbf{k}}^{2}}
$$

It is also convenient to further define a variable $q^{2}$ as:

$$
q^{2}=\overline{\mathbf{k}}^{2} L^{2} /(2 \pi)^{2}
$$

What Lüscher's formula tells us is a direct relation of $q^{2}$ and the elastic scattering phase shift $\tan \delta(q)$ in the infinite volume and it reads: 12,

$$
\tan \delta(q)=\frac{\pi^{3 / 2} q}{\mathscr{Z}_{00}\left(1 ; q^{2}\right)},
$$

where $\mathscr{Z}_{00}\left(1 ; q^{2}\right)$ is the zeta-function which can be evaluated numerically once its argument $q^{2}$ is given.

In the case of attractive interaction, the lowest two-particle energy level might be lower than the threshold resulting in $q^{2}<0$. When $q^{2}<0$, it is related to the phase $\sigma(q)$ via:

$$
\tan \sigma(q)=\frac{\pi^{3 / 2}(-i q)}{\mathscr{Z}_{00}\left(1 ; q^{2}\right)},
$$

where $(-i q)>0$ and the phase $\sigma(q)$ for pure imaginary $q$ is obtained from $\delta(q)$ by analytic continuation: $\tan \sigma(q)=-i \tan \delta(q)$ [12, 13]. The phase $\sigma(q)$ for pure imaginary $q$ is of physical significance since if there exists a true bound state at that particular energy, we have $\tan \sigma(q)=-1$ in the infinite volume and continuum limit. In the finite volume, the relation above is modified as: [13]

$$
\cot \sigma(q)=-1+\frac{6}{2 \pi \sqrt{-q^{2}}} e^{-2 \pi \sqrt{-q^{2}}}+\cdots
$$


where the finite-volume corrections are assumed to be small. Therefore, for $q^{2}<0$, we could compute $\tan \sigma(q)$ from Monte Carlo simulations and check the possibility of a bound state at that energy.

The above formulae apply to the case of a box with cubic symmetry. In real calculations, in order to have more accessible low-momentum modes, it is advantageous to use asymmetric volumes in the study of hadron scattering [14, 15, 16]. If the rectangular box is of size $L \times\left(\eta_{2} L\right) \times$ $\left(\eta_{3} L\right)$, then Eq. (2.3) is modified to:

$$
\tan \delta(q)=\frac{\pi^{3 / 2} q \eta_{2} \eta_{3}}{\mathscr{Z}_{00}\left(1 ; q^{2} ; \eta_{2}, \eta_{3}\right)},
$$

where the modified zeta-function $\mathscr{Z}_{00}\left(1, q^{2} ; \eta_{2}, \eta_{3}\right)$ is the analogue of $\mathscr{Z}_{00}\left(1 ; q^{2}\right)$ and its explicit definition can be found in Refs. [14, 15]. Similarly, for negative $q^{2}$, the formula is modified to:

$$
\tan \sigma(q)=\frac{\pi^{3 / 2}(-i q) \eta_{2} \eta_{3}}{\mathscr{Z}_{00}\left(1 ; q^{2} ; \eta_{2}, \eta_{3}\right)}
$$

Close to the scattering threshold, the phase has the following expansion:

$$
\frac{k}{\tan \delta(k)}=\frac{1}{a_{0}}+\frac{1}{2} r_{0} k^{2}+\cdots
$$

where $a_{0}$ is the scattering length and $r_{0}$ is the effective range.

\subsection{The operators and correlators}

To access the on-particle and two-particle energies for the $D^{*}$ and $\bar{D}_{1}$ systems, we need corresponding interpolating fields:

$$
Q_{i}(x)=\left[\bar{d} \gamma^{i} c\right](x), P_{i}(x)=\left[\bar{c} \gamma^{i} \gamma^{5} u\right](x)
$$

where $Q_{i}(x)$ stands for $D^{*}(2010)^{+}$while $P_{i}(x)$ stands for $\bar{D}_{1}(2420)^{0}$ and $i=1,2,3$ being the index to specify different spatial components.

A single-particle state with definite three-momentum $\mathbf{k}$ is represented by the Fourier transform of the above operators: which will be denoted as $Q_{i}(t, \mathbf{k})$ and $P_{i}(t, \mathbf{k})$ respectively. With the operators, one-particle correlation functions $C^{Q}(t, \mathbf{k})$ and $C^{P}(t, \mathbf{k})$ for the $D^{*+}$ and $\bar{D}_{1}^{0}$ mesons are constructed. In the large temporal separation limit, the energy $E(\mathbf{k})$ of a single meson with definite three-momentum $\mathbf{k}$ can be extracted from the effective mass plateau of the corresponding correlation functions as usual.

For the two-particle system formed by a $D^{*}$ and a $\bar{D}_{1}$ meson, the quantum number $J^{P}$ of the two-particle system can be: $J^{P}=0^{-}, 1^{-}, 2^{-}$. On the lattice, the rotational symmetry group $S O(3)$ is broken down to the corresponding point group. In our case, in order to access more non-degenerate low-momentum modes, it would be advantageous to use asymmetric box. This is particularly useful for scattering processes, as advocated in Ref. [16]. Following this strategy, we have adopted a rectangular box of size $L \times\left(\eta_{2} L\right) \times\left(\eta_{3} L\right)$ with $\eta_{2}=1$ and $\eta_{3} \neq 1$. In this case, the rotational group in the continuum is broken down to the basic point group $D_{4}$. In what follows, we will construct operators that transform according to different irreducible representations of the 
$D_{4}$ group which has four one-dimensional irreducible representations: $A_{1}, A_{2}, B_{1}, B_{2}$ and one two-dimensional irreducible representation: $E$. The following decomposition rules then apply:

$$
\mathbf{0}=A_{1}, \mathbf{1}=E \oplus A_{2}, \mathbf{2}=A_{1} \oplus B_{1} \oplus B_{2} \oplus E .
$$

Now, we consider the vector space $\left\{Q_{1}, Q_{2}, Q_{3}\right\} \otimes\left\{P_{1}, P_{2}, P_{3}\right\}$, which is 9-dimensional. Using standard group-theoretical methods, it is easy to find out that this 9-dimensional vector space is made up of two copies of $A_{1}$, one copy of $A_{2}, B_{1}$ and $B_{2}$ each and two copies of $E$. The basis operators of these irreps can be constructed and we found from our calculation that only one of the $A_{1}$ channels yields definite signal. In what follows, we will only focus on this particular channel for which the two-particle operator reads:

$$
\begin{aligned}
O^{\left(A_{1}\right)(1)}(t) & =\sum_{R \in G}\left[Q_{1}(t+1,-R \circ \mathbf{k}) P_{1}(t, R \circ \mathbf{k})+Q_{2}(t+1,-R \circ \mathbf{k}) P_{2}(t, R \circ \mathbf{k})\right. \\
& \left.+Q_{3}(t+1,-R \circ \mathbf{k}) P_{3}(t, R \circ \mathbf{k})\right],
\end{aligned}
$$

where $\mathbf{k}$ is a chosen three-momentum mode and $G$ is the group $D_{4}$ and $R \in G$ is an element of the group. Note that in the above definitions we have not included orbital angular momentum of the two-particles. Therefore we are only studying the $s$-wave scattering of the two mesons. This is sufficient for this particular case since near the threshold, the scattering is always dominated by $s$-wave contributions. The corresponding correlation matrix is given by:

$$
C_{m n}^{\left(A_{1}\right)(1)}(t)=\left\langle O_{m}^{\left(A_{1}\right)(1) \dagger}(t) O_{n}^{\left(A_{1}\right)(1)}(0)\right\rangle
$$

where $m$ and $n$ are indices for different momentum modes.

Two tricks can be utilized to calculate the correlation functions more efficiently. One is that the summation over $R \in D_{4}$ in the definition of the two-particle operator in Eq. (2.11) can be absorbed into the definition of the source when solving for the quark propagators. Another trick is that the light quark propagators are needed for the zero momentum mode only. Implementing these tricks, the final result of correlation function according for the operator $O^{\left(A_{1}\right)(1)}(t)$ is as follows:

$$
\begin{aligned}
C_{m n}^{\left(A_{1}\right)(1)}(t)= & \sum_{R \in G} \sum_{i, j=1}^{3}\left[\sum_{\mathbf{x}} e^{-i(R \circ \mathbf{p}) \cdot \mathbf{x}} \cdot\left(\gamma_{i} \gamma_{5}\right)_{\sigma \delta} \cdot\left(\gamma_{j} \gamma_{5}\right)_{\alpha^{\prime} \rho^{\prime}} \cdot X_{\delta b \mathbf{x} t+1}^{(d)\left(\alpha^{\prime} a^{\prime} 1\right)} \cdot\left(\sum_{R^{\prime} \in G} X_{\sigma b \mathbf{x} t+1}^{(c)\left(\rho^{\prime} a^{\prime} 1\right)}\left(R^{\prime} \circ \mathbf{q}\right)\right)^{*}\right] \\
& \cdot\left[\sum_{\mathbf{y}} e^{i(R \circ \mathbf{p}) \cdot \mathbf{y}} \cdot\left(\gamma_{i}\right)_{\rho \beta} \cdot\left(\gamma_{j}\right)_{\gamma^{\prime} \sigma^{\prime}} \cdot\left(\sum_{R^{\prime \prime} \in G} X_{\beta a \mathbf{y} t}^{(c)\left(\gamma^{\prime} b^{\prime} 0\right)}\left(R^{\prime \prime} \circ \mathbf{q}\right)\right) \cdot\left(X_{\rho a \mathbf{y} t}^{(u)\left(\sigma^{\prime} b^{\prime} 0\right)}\right)^{*}\right],
\end{aligned}
$$

with $m$ and $n$ being momentum mode indices with corresponding three-momenta $\mathbf{p}$ and $\mathbf{q}$, respectively; $R \in G$ being a group element of $D_{4} ; X$ being the quark propagators with appropriate sources.

\subsection{The effective mass}

For each symmetry channel, we have studied 5 different non-zero momentum modes in addition to the zero-momentum mode. To extract the two-particle energy eigenvalues, we adopt the usual Lüscher - Wolff method [1]. For this purpose, a new matrix $\Omega\left(t, t_{0}\right)$ is defined as:

$$
\Omega\left(t, t_{0}\right)=C\left(t_{0}\right)^{-\frac{1}{2}} C(t) C\left(t_{0}\right)^{-\frac{1}{2}},
$$




\begin{tabular}{|c|c|c|c|}
\hline & $\beta=2.5$ & $\beta=2.8$ & $\beta=3.2$ \\
\hline \hline$N_{\text {conf }}$ & 700 & 500 & 200 \\
\hline$a_{s}(\mathrm{fm})$ & 0.2037 & 0.1432 & 0.0946 \\
\hline lattice & $8 \times 8 \times 12 \times 40$ & $12 \times 12 \times 20 \times 64$ & $16 \times 16 \times 24 \times 80$ \\
\hline$\kappa_{\text {max }}^{c}$ & 0.0577 & 0.0598 & 0.0595 \\
\hline$\kappa_{\text {max }}^{\text {ud }}$ & 0.0613 & 0.0611 & 0.0606 \\
\hline
\end{tabular}

Table 1: Simulation parameters in this study. All lattices have the same aspect ratio: $\xi=5$.

where $t_{0}$ is a reference time-slice. Normally one picks a $t_{0}$ such that the signal is good and stable. The energy eigenvalues for the two-particle system are then obtained by diagonalizing the matrix $\Omega\left(t, t_{0}\right)$. The $i$-th eigenvalue of the matrix has the following behavior in the large $\left(t-t_{0}\right)$ limit:

$$
\lambda_{i}\left(t, t_{0}\right) \propto e^{-E_{i}\left(t-t_{0}\right)} .
$$

Therefore, the exact energy $E_{i}$ can be extracted from the effective mass plateau of the eigenvalue $\lambda_{i}$.

The real signal for the eigenvalue in our simulation turns out to be so noisy that reliable plateau cannot be found directly. Therefore, the following ratio was attempted:

$$
\mathscr{R}\left(t, t_{0}\right)=\frac{\lambda_{i}\left(t, t_{0}\right)}{C_{D^{*}}(t) C_{D_{1}}(t)} \propto e^{-\delta E_{i} \cdot t}
$$

where $C_{D^{*}}(t)$ and $C_{D_{1}}(t)$ are one-particle correlation function with zero momentum for the corresponding mesons. By taking this ratio, the signal to noise ratio is greatly enhanced. The energy difference $\delta E_{i}$ can be extracted reliably from the following effective mass:

$$
\delta E_{i}=E_{i}-m_{D^{*}}-m_{D_{1}}=\ln \left(\frac{\mathscr{R}(t)}{\mathscr{R}(t+1)}\right) .
$$

\section{Simulation details and results}

The gauge action use in this study is the tadpole improved gauge action on anisotropic lattices [17, 18, 19]. Quenched gauge field configurations are generated using the conventional Cabbibo-Mariani pseudo-heat bath algorithm with over-relaxation. Quark propagators are obtained using the so-called Multi-mass Minimal Residual $\left(\mathrm{M}^{3} \mathrm{R}\right)$ algorithm, which can yield the propagators with different quark masses at one inversion [22]. Dirichlet boundary conditions are used in the temporal direction for the fermion fields. Error estimates are made using the conventional jackknife method for all quantities. All the relevant simulation parameters are summarized in Table 1 . Some of the parameters have been obtained or tuned in Ref. [23, 21].

From the single-particle correlation functions, both with zero and with non-zero three-momenta, we have checked that the masses and dispersion relations for the single particle states of $D^{*}$ and $\bar{D}_{1}^{0}$. With Lüscher's formalism, we obtain the scattering phase shifts, which are fitted near the threshold to obtain the scattering length and the effective range in $A_{1}$ channel. After charm quark mass interpolations, chiral extrapolations and continuum extrapolations (for $a_{0}$ and $r_{0}$ ), the results are shown in Table \& 


\begin{tabular}{|c|c|c|c|c|}
\hline$\beta$ & $q^{2}$ & $\cot \sigma\left(q^{2}\right)$ & $a_{0}$ & $r_{0}$ \\
\hline 2.5 & $-0.026(0.003)$ & $5.23(0.65)$ & \multirow{3}{*}{$2.53(0.47) \mathrm{fm}$} & \multirow{3}{*}{$0.70(0.10) \mathrm{fm}$} \\
\hline 2.8 & $-0.064(0.005)$ & $0.16(0.18)$ & & \\
\hline 3.2 & $-0.053(0.016)$ & $0.92(0.93)$ & & \\
\hline
\end{tabular}

Table 2: Results for the lowest $q^{2}$ and the corresponding values for $\cot \sigma(q)$ as given by Eq. (2.2) and Eq. 2.7) and $a_{0}$ and $r_{0}$ as given by Eq. (2.8). Corresponding errors for the quantities are also given in the parenthesis.

To explore the possibility of a bound state, we recall that for a bound state to exist, $q^{2}$ has to be negative and in fact $q^{2} \rightarrow-\infty$ as $L \rightarrow \infty$. This results in the condition: $\cot \sigma(q)=-1$ as discussed in the subsection 2.1, Eq. (2.7). On the other hand, a scattering state will have: $q^{2} \simeq(1 / L)$ as $L \rightarrow \infty$. Results for the lowest (negative) $q^{2}$ and the corresponding values of $\cot \sigma(q)$ as computed from Eq. (2.7) are listed in Table2. It is seen that our results for $\cot \sigma(q)$ for the lowest (negative) $q^{2}$ are all positive. The absolute values for the lowest $q^{2}$ are also not large, not in favor of a shallow bound state.

One could investigate this possibility from the values of scattering length and effective range. Since we are studying the scattering near the threshold, it is appropriate to study the problem using non-relativistic quantum mechanics. Within non-relativistic quantum mechanics, it is known that, if the potential acquires an infinitely shallow bound state, the scattering length should approach negative infinity [13]. Our lattice results for the scattering lengths indicate that it is quite large but positive. This usually happens when the potential is on the verge of developing a shallow bound state.

If we further approximate the potential by a square-well potential, we could even estimate the depth $V_{0}$ and the range of the potential $R$ from our lattice results on $a_{0}$ and $r_{0}$. We find that, $R=r_{0}=0.70(10) \mathrm{fm}$ and $V_{0}=73(21) \mathrm{MeV}$. These values for a square-well potential also gives no bound states. If we fix $r_{0}=R=0.7 \mathrm{fm}$, the first bound state will occur at about $V_{0} \simeq 92 \mathrm{MeV}$.

\section{Conclusions}

In this paper, we present our quenched anisotropic lattice study for the scattering of $D^{*}$ and $\bar{D}_{1}$ mesons near the threshold. Our study focuses on the $s$-wave scattering in the channel $J^{P}=0^{-}$ and the scattering threshold parameters. After the chiral and continuum extrapolations, we obtain: $a_{0}=2.53(47) \mathrm{fm}$ and $r_{0}=0.70(10) \mathrm{fm}$, indicating that the interaction between a $D^{*}$ and a $\bar{D}_{1}$ meson is attractive in this channel. Based on our results for the the threshold parameters, it is unlikely that they form a genuine bound state right below the threshold in the channel $J^{P}=0^{-}$. The lowest two-particle state is likely to be a scattering state. This result might shed some light on the nature of the recently discovered $Z^{+}(4430)$ state by Belle. To further clarify the nature of the structure $Z^{+}(4430)$, lattice studies in other symmetry channels and preferably with dynamical fermions are much welcomed.

\section{Acknowledgments}

The author would like to thank Prof. H. Q. Zheng, Prof. S. H. Zhu and Prof. S. L. Zhu from 
Peking University for valuable discussions. This work is supported in part by NSFC under grant No.10835002, No.10675005 and No.10721063.

\section{References}

[1] S. K. Choi et al., Phys. Rev. Lett., 100:142001, 2008.

[2] Jonathan L. Rosner, Phys. Rev., D76:114002, 2007.

[3] Xiang Liu et al., Phys. Rev., D77:034003, 2008; Phys. Rev., D77:094015, 2008.

[4] D. V. Bugg. J. Phys., G35:075005, 2008.

[5] Cong-Feng Qiao, J. Phys., G35:075008, 2008.

[6] Su Houng Lee et al., Phys. Lett., B661:28-32, 2008.

[7] Eric Braaten and Meng Lu, Phys. Rev., D79:051503, 2009.

[8] Xiao-Hai Liu, Qiang Zhao, and Frank E. Close, Phys. Rev., D77:094005, 2008.

[9] Stephen Godfrey and Stephen L. Olsen, Ann. Rev. Nucl. Part. Sci., 58:51-73, 2008.

[10] G.Z. Meng et al., Phys. Rev. D, 80:034503, 2009.

[11] M. Lüscher, Commun. Math. Phys., 104:177, 1986; Commun. Math. Phys., 105:153, 1986; Nucl. Phys. B, 339:222, 1990;

[12] M. Lüscher, Nucl. Phys. B, 354:531, 1991; Nucl. Phys. B, 364:237, 1991.

[13] Shoichi Sasaki and Takeshi Yamazaki, PoS, LAT2007:131, 2007.

[14] X. Li and C. Liu, Phys. Lett. B, 587:100, 2004.

[15] X. Feng, X. Li, and C. Liu, Phys. Rev. D, 70:014505, 2004.

[16] Xin Li et al., JHEP, 06:053, 2007.

[17] C. Morningstar and M. Peardon, Phys. Rev. D, 60:034509, 1999.

[18] C. Liu, Chinese Physics Letter, 18:187, 2001.

[19] Y. Chen et al., Phys. Rev. D, 73:014516, 2006.

[20] Junhua Zhang and C. Liu, Mod. Phys. Lett. A, 16:1841, 2001.

[21] Shiquan Su et al., Int. J. Mod. Phys. A, 21:1015, 2006.

[22] U. Glaessner et al., S hep-lat/9605008.

[23] Wei Liu et al., Mod. Phys. Lett. A, 21:2313, 2006. 